\title{
A new computation method for a staggered grid of 3D EM field conservative modeling
}

\author{
Elena Yu. Fomenko ${ }^{1}$ and Toru Mogi ${ }^{2}$ \\ ${ }^{1}$ Geoelectromagnetic Research Institute RAS, Troitsk, Moscow Region 142092, Russia \\ ${ }^{2}$ Institute of Seismology and Volcanology, Hokkaido University, Sapporo 060-0810, Japan
}

(Received January 9, 2001; Revised August 8, 2001; Accepted September 21, 2001)

\begin{abstract}
A new three-dimensional (3D) MT modeling scheme conserving electric current and magnetic flux is developed. The scheme is based on finite difference (FD) staggered rectangular non-uniform grid formulation for the secondary electric field with continuous components of tangential electric and normal magnetic fields, in contrast to existing FD algorithms with a discontinuous E-field at the face of the cells. The scheme leads to a sparse 13-band complex symmetrical system of linear equations, which is effectively solved by fast and stable conjugate gradient (CG) methods. The preconditioning procedure was used to decrease the condition of a number of an ill-conditioned matrix system by several orders and stably and quickly solves the matrix system. The special module for the correction of divergence-free current $\mathbf{J}$ greatly increased the speed of convergence and accuracy, especially at low frequencies and for high-contrast resistivity or conductivity structures. A special procedure was developed to improve the accuracy of tangential magnetic and vertical electrical components at the Earth's surface and at the interface with a large conductivity contrast. The validity of the new algorithm was demonstrated for difficult models with high-contrast resistivity structures including topography and for COMMEMI project models.
\end{abstract}

\section{Introduction}

Much progress has been made in three-dimensional (3D) EM modeling with integral equation (IE), finite difference (FD), and finite element (FE) methods in the recent years (e.g. Wannamaker, 1991; Mackie et al., 1993; Alumbaugh et al., 1996; Smith, 1996; Avdeev et al., 1997; Weidelt, 1999; Xiong et al., 1999; Fomenko, 1999; Varentsov, 1999; and Sasaki, 1999). Models with an arbitrarily complex 3D resistivity distribution, including topography and high-resistivity contrast, require a large number of grid nodes. These models are especially difficult for using IE methods due to a full matrix system of order $N^{3}$ ( $N$ : number of nodes), thus these problems are mainly solved by FD or FE schemes. Known FD and FE schemes are incomplete for solving such difficult problems and thus require improvement. The following issues should be addressed: good accuracy over a wide range of frequencies, non-monotonic behavior of the residual, causing uncertainty of termination criteria, speed of convergence and stability of the numerical scheme. Thus, special attention has been devoted to schemes which obey the conservation laws of electric current and magnetic flux in a frequency domain for quasi-stationary electromagnetic fields. This approach leads to success in staggered finite difference (SFD) schemes based on magnetic fields (Mackie et al., 1993) and on electric fields (Smith, 1996; Alumbaugh et al., 1996; Weidelt, 1999; Xiong et al., 1999).

This paper describes a new step in the development of an electric-field solution leading to a fast, accurate, economi-

Copy right (C) The Society of Geomagnetism and Earth, Planetary and Space Sciences (SGEPSS); The Seismological Society of Japan; The Volcanological Society of Japan; The Geodetic Society of Japan; The Japanese Society for Planetary Sciences. cal, and conservative SFD scheme for wide-band frequencies and high-contrast structures. Our SFD scheme is based on an electric secondary field with continuous components at a staggered grid.

\section{Algorithm}

\subsection{Approximation and conservation laws}

First, we introduce a rectangular, non-uniform 'Normal Grid' with coordinates of the nodes $x_{i}, y_{j}, z_{k}$ and grid cells $\Delta_{i}=x_{i+1}-x_{i}, \Delta_{j}=y_{j+1}-y_{j}, \Delta_{k}=z_{k+1}-z_{k}$. We also define a 'Central Grid' with coordinates of the nodes $x_{i}^{\prime}=\left(x_{i}+x_{i+1}\right) / 2, y_{j}^{\prime}=\left(y_{j}+y_{j+1}\right) / 2, z_{k}^{\prime}=\left(z_{k}+z_{k+1}\right) / 2$ and grid cells $\Delta_{i}^{\prime}=\left(\Delta_{i}+\Delta_{i+1}\right) / 2, \Delta_{j}^{\prime}=\left(\Delta_{j}+\Delta_{j+1}\right) / 2$, $\Delta_{k}^{\prime}=\left(\Delta_{k}+\Delta_{k+1}\right) / 2$. Based on these grids, we construct a staggered grid (Fig. 1). The electric field (E) is taken to be at the center of the prism edges, and the magnetic field $(\mathbf{H})$ is taken to be at the center of the interfaces. Thus, $\mathbf{E}$ is always tangential and $\mathbf{H}$ is always normal to the conductivity and both are always continuous at the staggered grid.

Assuming the time harmonic dependence $\mathbf{e}^{-i \omega t}(\omega$ : angular frequency) and ignoring the displacement current in magnetotelluric modeling, Maxwell's equations for the total electric field (E) and magnetic field $(\mathbf{H})$ in a staggered grid lead to

$$
\begin{aligned}
& \oint \mathbf{H} d \mathbf{l}^{\prime}=\iint \sigma \mathbf{E} d \mathbf{s}^{\prime}, \\
& \oint \mathbf{E} d \mathbf{l}=i \omega \mu \iint \mathbf{H} d \mathbf{s}, \\
& \oint_{d V_{i j k}}(\sigma \mathbf{E}) d \mathbf{s}=0, \quad \text { or } \quad \operatorname{div}(\sigma \mathbf{E})=0,
\end{aligned}
$$




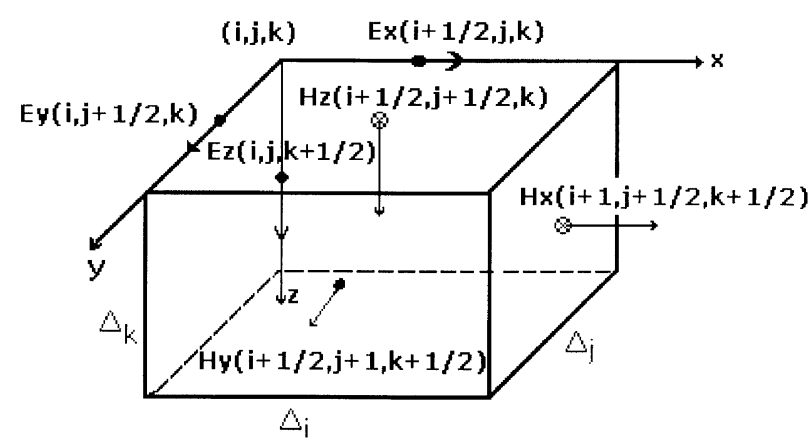

Fig. 1. A staggered grid cell $V(i, j, k)$. Tangential electric and normal magnetic field components are sampled at the center of edges $(\mathbf{E})$ and at the center of interfaces $(\mathbf{H})$, all of them are continuous. $\Delta_{i}, \Delta_{j}, \Delta_{k}$ denote the sizes of the cells.

$$
\oint_{d V_{i j k}}(\mathbf{H}) d \mathbf{s}=0, \quad \text { or } \quad \operatorname{div} \mathbf{H}=0,
$$

where $\mu$ is the magnetic permeability and $\sigma$ is the conductivity distribution in the cells of a normal grid.

We will use the formulation for secondary fields because it usually provides more stable and accurate solutions than formulations for total fields. The primary fields $\mathbf{E}^{p}, \mathbf{H}^{p}$ are selected to satisfy (1)-(4) for a model with primary conductivity $\sigma_{p}$. For the secondary fields $\mathbf{E}^{s}=\mathbf{E}-\mathbf{E}^{p}$, $\mathbf{H}^{s}=\mathbf{H}-\mathbf{H}^{p}$ and the secondary current $\mathbf{j}^{s}=\left(\sigma-\sigma_{p}\right) \mathbf{E}^{p}$, we have

$$
\begin{aligned}
\oint \mathbf{H}^{s} d \mathbf{l}^{\prime} & =\iint\left(\sigma \mathbf{E}^{s}+\left(\sigma-\sigma_{p}\right) \mathbf{E}^{p}\right) d \mathbf{s}^{\prime} \\
& =\iint\left(\sigma \mathbf{E}^{\mathbf{s}}+\mathbf{j}^{\mathbf{s}}\right) d \mathbf{s}^{\prime}, \\
\oint \mathbf{E}^{s} d \mathbf{l} & =i \omega \mu \iint \mathbf{H}^{s} d \mathbf{s},
\end{aligned}
$$

where $d \mathbf{l}, d \mathbf{s}$ correspond to the normal grid cells and $d \mathbf{l}^{\prime}, d \mathbf{s}^{\prime}$ to the central grid cells.

$$
\begin{aligned}
& d \mathbf{s}_{\mathbf{x} i j k}=\Delta_{j-1} \Delta_{k-1} \mathbf{n}_{\mathbf{x}}, \quad d \mathbf{s}_{\mathbf{y}_{i j k}}=\Delta_{k-1} \Delta_{j-1} \mathbf{n}_{\mathbf{y}}, \\
& d \mathbf{s}_{\mathbf{z} i j k}=\Delta_{i-1} \Delta_{j-1} \mathbf{n}_{z}, \\
& d \mathbf{l}_{\mathbf{x}}=\Delta_{i-1} \mathbf{n}_{i}, \quad d \mathbf{l}_{\mathbf{y}}=\Delta_{j-1} \mathbf{n}_{j}, \quad d \mathbf{l}_{\mathbf{z}}=\Delta_{k-1} \mathbf{n}_{k},
\end{aligned}
$$

$d \mathbf{s}_{\mathbf{x}}^{\prime}, d \mathbf{s}_{\mathbf{y}}^{\prime}, d \mathbf{s}_{\mathbf{z}}^{\prime}$ and $d \mathbf{l}_{\mathbf{x}}^{\prime}, d \mathbf{l}_{\mathbf{y}}^{\prime}, d \mathbf{l}_{\mathbf{z}}^{\prime}$ are given by the same equations with primes (e.g. $d \mathbf{s}_{\mathbf{x} i j k}^{\prime}=\Delta_{j-1}^{\prime} \Delta_{k-1}^{\prime} \mathbf{n}_{\mathbf{x}}$ ). $\mathbf{n}_{\mathbf{x}}, \mathbf{n}_{\mathbf{y}}, \mathbf{n}_{\mathbf{z}}$ are unit vectors correspondingly along the $x, y, z$ directions.

After staggered finite difference (SFD) approximation of Eqs. (1) and (2), we obtain six scalar equations shown as (5) and (6):

$$
\begin{aligned}
& \left(\mathbf{H}_{j+1 / 2}^{z}-\mathbf{H}_{j-1 / 2}^{z}\right) \Delta_{k-1}^{\prime}-\left(\mathbf{H}_{k+1 / 2}^{y}-\mathbf{H}_{k-1 / 2}^{y}\right) \Delta_{j-1}^{\prime} \\
& \quad=\langle\sigma\rangle_{i, j-1, k-1}^{x} \mathbf{E}_{i-1 / 2, j, k}^{x} \Delta_{j-1}^{\prime} \Delta_{k-1}^{\prime} \\
& \left(\mathbf{H}_{k+1 / 2}^{x}-\mathbf{H}_{k-1 / 2}^{x}\right) \Delta_{i-1}^{\prime}-\left(\mathbf{H}_{i+1 / 2}^{z}-\mathbf{H}_{i-1 / 2}^{z}\right) \Delta_{k-1}^{\prime} \\
& \quad=\langle\sigma\rangle_{i-1, j, k-1}^{y} \mathbf{E}_{i, j-1 / 2, k}^{y} \Delta_{k-1}^{\prime} \Delta_{i-1}^{\prime} \\
& \left(\mathbf{H}_{i+1 / 2}^{y}-\mathbf{H}_{i-1 / 2}^{y}\right) \Delta_{j-1}^{\prime}-\left(\mathbf{H}_{j+1 / 2}^{x}-\mathbf{H}_{j-1 / 2}^{x}\right) \Delta_{i-1}^{\prime} \\
& \quad=\langle\sigma\rangle_{i-1, j-1, k}^{z} \mathbf{E}_{i, j, k-1 / 2}^{z} \Delta_{i-1}^{\prime} \Delta_{j-1}^{\prime} \\
& \left(\mathbf{E}_{j}^{z}-\mathbf{E}_{j-1}^{z}\right) \Delta_{k-1}-\left(\mathbf{E}_{k}^{y}-\mathbf{E}_{k-1}^{y}\right) \Delta_{j-1} \\
& \quad=i \omega \mu \mathbf{H}_{i, j-1 / 2, k-1 / 2}^{x} \Delta_{j-1} \Delta_{k-1}
\end{aligned}
$$

$$
\begin{gathered}
\left(\mathbf{E}_{k}^{x}-\mathbf{E}_{k-1}^{x}\right) \Delta_{i-1}+\left(\mathbf{E}_{i}^{z}-\mathbf{E}_{i-1}^{z}\right) \Delta_{k-1} \\
\quad=i \omega \mu \mathbf{H}_{i-1 / 2, j, k-1 / 2}^{y} \Delta_{k-1} \Delta_{i-1}, \\
\left(\mathbf{E}_{i}^{y}-\mathbf{E}_{i-1}^{y}\right) \Delta_{j-1}-\left(\mathbf{E}_{j}^{x}-\mathbf{E}_{j-1}^{x}\right) \Delta_{i-1} \\
\quad=i \omega \mu \mathbf{H}_{i-1 / 2, j-1 / 2, k}^{z} \Delta_{i-1} \Delta_{j-1},
\end{gathered}
$$

where, for example,

$$
\begin{aligned}
\langle\sigma\rangle_{i-1, j-1, k}^{z}=[ & \sigma_{i, j, k} \Delta_{i} \Delta_{j}+\sigma_{i-1, j, k} \Delta_{i-1} \Delta_{j} \\
& +\sigma_{i, j-1, k} \Delta_{i} \Delta_{j-1} \\
& \left.+\sigma_{i-1, j-1, k} \Delta_{i-1} \Delta_{j-1}\right] /\left(4 \Delta_{i-1}^{\prime} \Delta_{j-1}^{\prime}\right) .
\end{aligned}
$$

The original system describing (5), (6) is a pair of coupled first-order equations for $\mathbf{E}$ and $\mathbf{H}$. In order to decrease the number of equations, we eliminate $\mathbf{H}$-field from Eq. (5) using the right-hand side of Eq. (6). The second-order SFD equation was introduced as follows:

$$
\widetilde{\mathbf{A}}\left(\mathbf{E}^{s}+\mathbf{E}^{p}\right)=\widetilde{\mathbf{A}} \mathbf{E}^{\text {total }}=0 .
$$

The matrix $\widetilde{\mathbf{A}}$ is neither Hermitian nor symmetric, but it can be transformed to the complex symmetric matrix $\hat{\mathbf{A}}$ by multiplying the obtained equations by factor $\Delta_{i}$ for $\mathbf{E}^{x}$ members in the right side, $\Delta_{j}$ for $\mathbf{E}^{y}$ and $\Delta_{k}$ for $\mathbf{E}^{z}$.

We introduce SFD operators $\nabla_{s f d}, \operatorname{div}_{s f d}, \nabla_{s f d} \times \mathbf{F}$ at the staggered grid as

$$
\begin{aligned}
\left(\nabla_{s f d} \mathbf{F}\right)_{i j k}=\{ & \left(\mathbf{F}_{i}^{x}-\mathbf{F}_{i-1}^{x}\right) / \Delta_{i-1}^{\prime},\left(\mathbf{F}_{j}^{y}-\mathbf{F}_{j-1}^{y}\right) / \Delta_{j-1}^{\prime} \\
& \left.\left(\mathbf{F}_{k}^{z}-\mathbf{F}_{k-1}^{z}\right) / \Delta_{k-1}^{\prime}\right\} \\
\left(\operatorname{div}_{s f d} \mathbf{F}\right)_{i j k}= & \left(\mathbf{F}_{i}^{x}-\mathbf{F}_{i-1}^{x}\right) / \Delta_{i-1}^{\prime}+\left(\mathbf{F}_{j}^{y}-\mathbf{F}_{j-1}^{y}\right) / \Delta_{j-1}^{\prime} \\
& +\left(\mathbf{F}_{k}^{z}-\mathbf{F}_{k-1}^{z}\right) / \Delta_{k-1}^{\prime} \\
\left(\nabla_{s f d} \times \mathbf{F}\right)_{z, i j k}= & \left(\mathbf{F}_{i}^{y}-\mathbf{F}_{i-1}^{y}\right) / \Delta_{i-1} \\
& \quad-\left(\mathbf{F}_{j}^{x}-\mathbf{F}_{j-1}^{x}\right) / \Delta_{j-1}, \quad \text { etc. }
\end{aligned}
$$

After moving the primary field $\mathbf{E}^{p}$ to the right-hand side, we obtain the final complex symmetric linear system for the secondary electric field $\mathbf{E}^{s}$ with second-order SFD matrix operators $\hat{\mathbf{A}}, \hat{\mathbf{A}}^{p}$ and right-hand side vector $\mathbf{b}$

$$
\hat{\mathbf{A}} \mathbf{E}^{s}=-\hat{\mathbf{A}} \mathbf{E}^{p}=\mathbf{b}=\mathbf{f}-\hat{\mathbf{A}}^{p} \mathbf{E}^{p}
$$

or

$$
\begin{aligned}
\left(\begin{array}{lll}
\mathbf{A}_{x x} & \mathbf{A}_{x y} & \mathbf{A}_{x z} \\
\mathbf{A}_{y x} & \mathbf{A}_{y y} & \mathbf{A}_{y z} \\
\mathbf{A}_{z x} & \mathbf{A}_{z y} & \mathbf{A}_{z z}
\end{array}\right)\left[\begin{array}{c}
\mathbf{E}^{x, s} \\
\mathbf{E}^{y, s} \\
\mathbf{E}^{z, s}
\end{array}\right] \\
=\left[\begin{array}{c}
f^{x} \\
f^{y} \\
f^{z}
\end{array}\right]-\left(\begin{array}{ccc}
\mathbf{A}_{x x}^{p} & \mathbf{A}_{x y}^{p} & \mathbf{A}_{x z}^{p} \\
\mathbf{A}_{y x}^{p} & \mathbf{A}_{y y}^{p} & \mathbf{A}_{y z}^{p} \\
\mathbf{A}_{z x}^{p} & \mathbf{A}_{z y}^{p} & \mathbf{A}_{z z}^{p}
\end{array}\right)\left[\begin{array}{l}
\mathbf{E}^{x, p} \\
\mathbf{E}^{y, p} \\
\mathbf{E}^{z, p}
\end{array}\right]=\left[\begin{array}{c}
b^{x} \\
b^{y} \\
b^{z}
\end{array}\right],
\end{aligned}
$$

where

$$
\begin{aligned}
& \hat{\mathbf{A}}=\left(\nabla_{s f d} \times \nabla_{s f d}-i \omega \mu \sigma \mathbf{I}\right) d v^{\prime}, \\
& \hat{\mathbf{A}}^{p}=\left(\nabla_{s f d} \times \nabla_{s f d}-i \omega \mu \sigma_{p} \mathbf{I}\right) d v^{\prime}, \\
& f^{x}=i \omega \mu\left\langle\sigma-\sigma^{p}\right\rangle_{i, j-1, k-1}^{x} \Delta_{i} \Delta_{j-1}^{\prime} \Delta_{k-1}^{\prime} \mathbf{E}_{i-1 / 2, j, k}^{x, p}, \\
& f^{y}=i \omega \mu\left\langle\sigma-\sigma^{p}\right\rangle_{i-1, j, k-1}^{y} \Delta_{i-1}^{\prime} \Delta_{j} \Delta_{k-1}^{\prime} \mathbf{E}_{i, j-1 / 2, k}^{y, p}, \\
& f^{z}=i \omega \mu\left\langle\sigma-\sigma^{p}\right\rangle_{i-1, j-1, k}^{z} \Delta_{i-1}^{\prime} \Delta_{j-1}^{\prime} \Delta_{k} \mathbf{E}_{i, j, k-1 / 2}^{z, p},
\end{aligned}
$$


I is the identity matrix.

The block operators $\mathbf{A}_{x x}, \mathbf{A}_{x y}, \mathbf{A}_{x z}$ can be written as

$$
\begin{aligned}
\mathbf{A}_{x x} \mathbf{E}^{x}= & \left\{\left[\left(\mathbf{E}_{k}^{x}-\mathbf{E}_{k+1}^{x}\right) / \Delta_{k}+\left(\mathbf{E}_{k}^{x}-\mathbf{E}_{k-1}^{x}\right) / \Delta_{k-1}\right]\right. \\
& \left./ \Delta_{k-1}^{\prime}\right\} \Delta_{i} \Delta_{j-1}^{\prime} \Delta_{k-1}^{\prime} \\
& +\left\{\left[\left(\mathbf{E}_{j}^{x}-\mathbf{E}_{j+1}^{x}\right) / \Delta_{j}+\left(\mathbf{E}_{j}^{x}-\mathbf{E}_{j-1}^{x}\right) / \Delta_{j-1}\right]\right. \\
& \left./ \Delta_{j-1}^{\prime}\right\} \Delta_{i} \Delta_{j-1}^{\prime} \Delta_{k-1}^{\prime} \\
& -i \omega \mu\langle\sigma\rangle_{i, j-1, k-1}^{x} \mathbf{E}_{i-1 / 2, j, k}^{x} \Delta_{i} \Delta_{j-1}^{\prime} \Delta_{k-1}^{\prime}, \\
\mathbf{A}_{x y} \mathbf{E}^{y}= & \left(\mathbf{E}_{i+1, j+1 / 2}^{y}-\mathbf{E}_{i+1, j-1 / 2}^{y}\right. \\
& \left.-\mathbf{E}_{i, j+1 / 2}^{y}+\mathbf{E}_{i, j-1 / 2}^{y}\right) \Delta_{k-1}^{\prime}, \\
\mathbf{A}_{x z} \mathbf{E}^{z}= & \left(\mathbf{E}_{i+1, k+1 / 2}^{z}-\mathbf{E}_{i+1, k-1 / 2}^{z}\right. \\
& \left.-\mathbf{E}_{i, k+1 / 2}^{z}+\mathbf{E}_{i, k-1 / 2}^{z}\right) \Delta_{j-1}^{\prime} .
\end{aligned}
$$

Other elements of the operator $\hat{\mathbf{A}}$ have similar forms.

Let us now consider the conservation laws describing (3) and (4) on the staggered grid. The SFD operators, introduced in (8.1)-(8.3), automatically provide the following vector equalities:

$$
\begin{aligned}
& \nabla_{s f d} \times(\nabla \phi)=0 \quad \text { for any SFD scalar } \phi, \\
& \operatorname{div}\left(\nabla_{s f d} \times \mathbf{F}\right)=0 \quad \text { for any SFD vector } \mathbf{F}, \\
& \nabla_{s f d} \times \nabla_{s f d} \times \mathbf{F}=-\nabla_{s f d}^{2} \mathbf{F}+\nabla_{s f d} \operatorname{div}_{s f d} \mathbf{F} \\
& \quad \text { for any SFD vector } \mathbf{F},
\end{aligned}
$$

and the following two SFD conservation laws on the staggered grid are introduced, when the div operator is applied to the original equations (5) and (6) for the total fields:

$$
\begin{aligned}
& \operatorname{div}_{s f d} \mathbf{j}^{\text {total }}=\operatorname{div}_{s f d}(\sigma \mathbf{E})=0, \\
& i \omega \mu \operatorname{div}_{s f d} \mathbf{H}=0 .
\end{aligned}
$$

Equations (11), (12) do not hold automatically when $\sigma=$ 0 (in the air) or $\omega=0$ (static case), as well as at low frequencies where computer round-off errors increase, even if double precision is used. Our transformation from the pair of coupled E-H equations of the first order (5), (6) to the final stand-alone second-order Eq. (9) for $\mathbf{E}$ field cannot automatically provide conservation laws (11), (12) in these cases, due to the elimination of matrix entries associated with regions where $\sigma=0$ in the $\mathbf{E}-\mathbf{H}$ formulation. In the regions where $\sigma=0$ (air or insulator layer/body in the earth), if $\mathbf{E}$ is an SFD solution, any $\mathbf{E}+\nabla \phi$ is also an SFD solution. Moreover, the final linear system (9) is ill-conditioned because the main part of its operator $\left(\mathbf{A}_{x x}, \mathbf{A}_{y y}, \mathbf{A}_{z z}\right)$ contains an ill-conditioned Laplace operator. In order to overcome these problems, we use $\sigma=10^{-8} \sim 10^{-10} \mathrm{~S} / \mathrm{m}$ instead of $\sigma=0$ and solve Eq. (9) for the $\mathbf{E}^{s}$-field together with Eq. (11), periodically making divergence-free corrections to the current $\mathbf{j}=\sigma \mathbf{E}$ to suppress computer round-off errors (see the details in Subsection 2.7).

At $\omega=0$, the electric field becomes curl-free, thus it can be expressed as $\mathbf{E}=\nabla \phi$. If we add Eq. (11), we will avoid uncertainty when calculating the electric field, because these two equations follow in the stationary equation $\Delta \phi=0$.

It is very important to note that solving Eq. (9) without the conservation law (11) as a stand-alone equation causes inaccurate calculations of the $\mathbf{E}$ and $\mathbf{H}$ fields, especially at low frequencies, in the vicinity of a dipole source (Alumbaugh et al., 1996), and for large grids containing 100,000 and more cells (the condition number of the matrix is more than $10^{8}$ ).

The $\mathbf{H}$-field is calculated using the curl-operator in the generalized Eq. (5) and (6) after solving Eq. (9) and (11) at the staggered grid (see Subsection 2.6). Due to the vector equality (10.2) and (6), the resulting magnetic field is automatically divergence-free with staggered grid and satisfies the conservation law in (12).

Using the Taylor series, it is easy to show that the proposed scheme has second-order approximation at the uniform grid $\left(\Delta_{i}=\Delta_{j}=\Delta_{k}\right)$ and first-order approximation in the area of non-uniform grid cells.

\subsection{Boundary conditions}

Equations (1)-(4) for the all $\mathbf{E}, \mathbf{H}$ components uniquely determine the current $\sigma \mathbf{E}$ and magnetic field $\mathbf{H}$ throughout the 3D model, when the tangential $\mathbf{E}$ or $\mathbf{H}$ components are specified at the boundary (Harrington, 1961).

In this study, we used simple boundary conditions (BC) for the tangential components of the secondary electric field $\mathbf{E}_{\text {tang }}^{s}$ at each vertical boundary surface;

$$
\mathbf{E}_{\text {tang }}^{s}=0 \text {. }
$$

If the modelling area has $2 \mathrm{D}$ anomalous bodies, we set

$$
\partial \mathbf{E} / \partial \mathbf{n}=0 \quad \text { or } \quad \partial \mathbf{E}^{s} / \partial \mathbf{n}=-\partial \mathbf{E}^{p} / \partial \mathbf{n}
$$

at the vertical plane, which is normal to the $2 \mathrm{D}$ prolongated body.

Along the top and bottom faces, bilinear interpolated horizontal fields from the four corner 1D sections are specified.

Asymptotic boundary conditions (ABC), considered to be more accurate, can be constructed for the $\mathbf{E}^{s}$ field at the staggered grid using a generalization of the approach offered by Zhdanov et al. (1982) and Spichak (1985). Taking into account the behavior of the electric field toward infinity, use of the $\mathrm{ABC}$ reduces the size of the $3 \mathrm{D}$ modeling area but, in our case, leads to the loss of symmetrical property of the matrix which requires us to store 13 bands of the matrix in the computer memory, instead of three bands in the symmetrical case. Moreover, the $\mathrm{ABC}$ require knowledge of the three components of the $\mathbf{E}$ field at the boundary. By this reason we did not use $\mathrm{ABC}$. We retain the simple $\mathrm{BC}$ (13) and (14), even for large grids (250,000 cells following in 750,000 equations). We overcame the issues using an effective preconditioning of the matrix and a fast solver, which are described below.

\subsection{Primary E-field}

In magnetotellurics, a primary field $\mathbf{E}^{p}$ is usually based on a $1 D$ normal section $\left(\mathbf{E}^{1 D}\right)$ or solution of a 2 D-simplified problem on the outer grid $\left(\mathbf{E}^{2 D}\right)$ (Mackie et al., 1993). The primary fields $\mathbf{E}^{p}, \mathbf{H}^{p}$ must satisfy (1)-(4) for a model with primary $\sigma_{p}$ conductivity distribution. In particular, when $1 \mathrm{D}$ normal sections are equal at both left and right sides, the member $\hat{\mathbf{A}}^{p} \mathbf{E}^{p}$ in the right-hand side of Eq. (9) equals zero. The member $\hat{\mathbf{A}}^{p} \mathbf{E}^{p}$ is not zero for other models due to the appearance of a secondary current at the conjunction of $1 \mathrm{D}$ sections.

\subsection{Preconditioning of the matrix}

The matrix operator $\hat{\mathbf{A}}$ in Eq. (9) is usually ill-conditioned. The main part of the operator contains an ill-condi- 
tioned Laplace operator. The condition number (cond $\hat{\mathbf{A}}$ ) usually takes a range of $10^{7} \sim 10^{14}$, which depends on the number of grid cells and the irregularity of the grid. Moreover, the matrix operator $\hat{\mathbf{A}}$ is indefinite because it contains $\nabla$ operator (see (10.3)). Thus, we have to make preconditioning before solving the linear system (9) in order to greatly diminish the condition number. We use effective ILU preconditioner (Implicit LU factorization by Cholesky) for the main blocks of $\mathbf{A}_{x x}, \mathbf{A}_{y y}, \mathbf{A}_{z z}$ only because the main part of the ill-conditioned matrix operator $\hat{\mathbf{A}}$ is concentrated in its main blocks. This operation decreases the condition number by several orders, resulting in a linear system with cond $\hat{\mathbf{A}}$ being not more than $10^{3}$, and greatly accelerates speed of convergence (Smith, 1996; Fomenko, 1999).

The original system $\hat{\mathbf{A}} \mathbf{E}^{s}=\mathbf{b}$ is replaced by a preconditioned system with appropriate $\mathbf{K}_{1}$ and $\mathbf{K}_{2}$ preconditioners:

$$
\left(\mathbf{K}_{1}^{-1} \hat{\mathbf{A}} \mathbf{K}_{2}^{-1}\right)\left(\mathbf{K}_{2} \mathbf{E}^{s}\right)=\mathbf{K}_{1}^{-1} \mathbf{b}
$$

where a preconditioned matrix $\mathbf{K}_{1}^{-1} \hat{\mathbf{A}} \mathbf{K}_{2}^{-1}$ is close enough to the identity matrix $\mathbf{I}$. The preconditioner $\mathbf{M}$ for the operator $\hat{\mathbf{A}}=\mathbf{L}+\mathbf{D}+\mathbf{U}$ is constructed by using Cholesky's ILU factorization of matrix $\hat{\mathbf{A}}$ :

$$
\mathbf{M}=(\tilde{\mathbf{D}}+\mathbf{L})(\tilde{\mathbf{D}})^{-1}(\tilde{\mathbf{D}}+\mathbf{U})
$$

where a new diagonal $\widetilde{\mathbf{D}}$ is calculated using the matrix diagonal $\mathbf{D}$, lower $\mathbf{L}$ and upper $\mathbf{U}$ triangular sub-matrices without diagonal D. The SSOR preconditioning, a simple particular case of LU preconditioning, uses matrix $\mathbf{D}$ instead of $\widetilde{\mathbf{D}}$ in (16). The simplest, but only rough, is the diagonal (or Jacobi) preconditioner $\mathbf{M}=\mathbf{D}^{-1}$, where $\mathbf{K}_{1}=\mathbf{K}_{2}=\mathbf{D}^{1 / 2}$. We used a right preconditioning scheme where $\mathbf{K}_{1}=\mathbf{I}$ and $\mathbf{K}_{2}=\mathbf{M}$ in this study.

\subsection{Solvers of the linear system}

A comprehensive study of the numerical effectiveness for a new advanced group of conjugate gradient iterative solvers BICGSTAB (Van der Vorst, 1992), BICGSTAB2 (Steijpen et al., 1994), QMRSTAB (Chan et al., 1994), Chebyshev's solver (Manteuffel et al., 1995) was numerically examined by Varentsov (1999) and Fomenko (1999). All of these CG methods belong to the group of three-step methods, which provide the global minimization of the residual within certain iterations and give fast quasi-monotonic convergence. This group of methods is essentially more stable and faster than the CG methods of the previous generation such as generalized minimal residuals algorithm (MRA-Mackie et al., 1993), BI-CG and its modifications (Smith, 1996; Weaver et al., 1999; Fomenko, 1999) or successive over relaxation method (SOR-Spichak, 1992). The latter methods belonged to the group of two-step methods which provides minimization of the residual at the current iteration only. More important, the new methods provide fast convergence for the difficult models with high resistivity contrasts and a large variety of grid cell sizes where the old methods (MRA, SOR) fail (Fomenko, 1999).

The criteria for the normalized $\|\mathbf{r}\| /\left\|\mathbf{r}_{\mathbf{o}}\right\|$ and preconditioned $\sqrt{\left\langle\mathbf{M}^{-1} \mathbf{r}, \mathbf{r}\right\rangle} /\left\|\mathbf{r}_{\mathbf{o}}\right\|$ residual $\mathbf{r}=\mathbf{b}-\hat{\mathbf{A}} \mathbf{E}^{s}$, as well as for the $D *|\operatorname{div} \mathbf{J}| /\|\mathbf{J}\|$ value ( $D$ : average size of the cells) and $\left\|\mathbf{E}^{n+1}-\mathbf{E}^{n}\right\| /\left\|\mathbf{E}^{n+1}\right\|$ (n: number of iteration) were usually achieved (1\% accuracy) in the first tens of iterations for grids of moderate size (30,000-60,000 cells) when QMRSTAB or BiCGSTAB solvers were used. These criteria were sometimes evaluated by $\left\|\mathbf{E}^{n}-\mathbf{E}^{\text {ref }}\right\| /\left\|\mathbf{E}^{\text {ref }}\right\|<\varepsilon$ in relation to the 'reference' solution which is calculated with maximum accuracy $\left(10^{-12}\right.$ and better) (Fomenko, 1999). A more accurate and reliable termination criterion was proposed by Varentsov (1999) for the magnetic field, and it has been transformed to the electric field in this study. The criterion is given by:

$$
\|\operatorname{del} \mathbf{E}\| /\left\|\mathbf{E}^{s}\right\|<\varepsilon
$$

where del $\mathbf{E}$ means the difference in $l_{2}$ norm between the $\mathbf{E}$ field before the divergence correction (18) and the corrected E field solution.

\subsection{Calculation of $\mathbf{H}$-field}

The $\mathbf{H}$ field is calculated using the curl-operator in Eq. (6) after solving Eqs. (9) and (11) at the staggered grid. Tangential electric and normal magnetic field components are continuous at the staggered grid due to our selection of grid nodes. It helps to stably calculate the magnetic $H_{z}$ component in the center of cell interfaces $x_{i+1 / 2}, y_{j+1 / 2}$ at each level $z=z_{k}$ by using formula (6.3). The horizontal components $H_{x}$ and $H_{y}$ are first calculated at the levels $z=z_{k}^{\prime}$ and $z=z_{k-1}^{\prime}$ by using formulae (6.1)-(6.2). Then we calculate $H_{x}$ and $H_{y}$ at $z=z_{k}$ by vertical linear interpolation, involving $H_{x}$ and $H_{y}$ values at $z=z_{k}^{\prime}, z=z_{k-1}^{\prime}$.

A special algorithm was derived to improve the accuracy of the horizontal magnetic components $H_{x}$ and $H_{y}$ at the surface level $z_{k_{0}}=0$ and at the interfaces $z_{k}$ with high-contrast of the conductivity/resistivity. This program does not use $\operatorname{rot} \mathbf{E}$ operator, where its derivative are not accurately calculated in the area of large conductivity contrasts. First, values of continuous components $E_{x}, E_{y}$ and $H_{z}$ are interpolated vertically to the level $z_{k-1 / 4}$ involving the corresponding values at $z_{k}$ and $z_{k-1}$. Then, instead of (5.1)-(5.2), we write modified formulae $\left(5.1^{\prime}\right)-\left(5.2^{\prime}\right)$ for the cell between $x_{i-1}$ and $x_{i}, y_{j-1}$ and $y_{j}, z_{k}$ and $z_{k-1 / 2}$

$$
\begin{aligned}
\left(\mathbf{H}_{i-1 / 2, j+1 / 2, k-1 / 4}^{z}-\mathbf{H}_{i-1 / 2, j-1 / 2, k-1 / 4}^{z}\right) \Delta_{k} / 2 & \\
& -\left(\mathbf{H}_{i-1 / 2 j, k}^{y}-\mathbf{H}_{i-1 / 2 j, k-1 / 2}^{y}\right) \Delta_{j-1}^{\prime} / 2 \\
= & \left(\sigma_{j-1} \Delta_{j-1}+\sigma_{j} \Delta_{j}\right) /\left(\Delta_{j-1}+\Delta_{j}\right) \\
& \cdot \mathbf{E}_{i-1 / 2, j, k-1 / 4}^{x} \Delta_{j-1}^{\prime} \Delta_{k} / 4 \\
\left(\mathbf{H}_{i, j-j-1 / 2, k}^{x}-\mathbf{H}_{i, j-1 / 2, k-1 / 2}^{x}\right) \Delta_{i-1}^{\prime} / 2 & \\
\quad & \left(\mathbf{H}_{i+1 / 2, j-1 / 2, k-1 / 4}^{z}-\mathbf{H}_{i-1 / 2, j-1 / 2, k-1 / 4}^{z}\right) \Delta_{k} / 2 \\
= & \left(\sigma_{i-1} \Delta_{i-1}+\sigma_{i} \Delta_{i}\right) /\left(\Delta_{i-1}+\Delta_{i}\right) \\
& \cdot \mathbf{E}_{i, j-1 / 2, k-1 / 4}^{y} \Delta_{i-1}^{\prime} \Delta_{k} / 4,
\end{aligned}
$$

which contain interpolated values $E_{x}, E_{y}$ and $H_{z}$ at a depth $z_{k-1 / 4}$, earlier calculated $H_{x}$ or $H_{y}$ at a depth $z_{k-1 / 2}$ and do not contain $E_{z}$. Components $H_{i, j-1 / 2, k}^{x}$ or $H_{i-1 / 2, j, k}^{y}$ are calculated at the level $z_{k}$ in the center of the edges by using formula $\left(5.2^{\prime}\right)$ or $\left(5.1^{\prime}\right)$. Finally, $H_{x}$ and $H_{y}$ components are interpolated horizontally from the center of the edges to the center of the cell interfaces $x_{i-1 / 2}, y_{j-1 / 2}, z_{k}$.

\subsection{Div $J$ correction and accuracy control during an iterative process}

The constructed SFD solution $\sigma \mathbf{E}$ is divergent-free in (11) at the area of modeling, but it loses this property in the area 
where $\sigma=0$ or $\omega=0$ and also at the low frequencies during the iterative process due to computer-rounding errors. Thus, following Smith (1996) and Mackie et al. (1993), we also make an adaptive correction of the E-field by adding a gradient of unknown SFD potential $\phi$ to the iterative solution $\mathbf{E}^{(N)}$ at the $N$ th iteration. The corrected electric field $\mathbf{E}^{(N, \text { cor })}=\mathbf{E}^{(N)}+\nabla \phi$ satisfies the equation,

$$
\operatorname{div} \mathbf{j}^{(N)}=\operatorname{div}\left(\sigma \mathbf{E}^{(N, \text { cor })}\right)=0=\operatorname{div} \sigma \mathbf{E}^{(N)}+\operatorname{div}(\sigma \nabla \phi) .
$$

The potential $\phi$ is calculated from the scalar SFD boundary problem:

$$
-\operatorname{div}(\sigma \nabla \phi)=\operatorname{div}\left(\sigma \mathbf{E}^{(N)}\right),
$$

with boundary condition $\left.\phi\right|_{b n d}=0$.

Equation (18) is numerically solved using termination criteria

$$
\left\|\operatorname{div}\left(\sigma \mathbf{E}^{(N)}+\nabla \phi\right)\right\| /\left\|\operatorname{div}\left(\sigma \mathbf{E}^{(N)}\right)\right\| \leq \varepsilon
$$

Numerical experiments demonstrated that $\varepsilon=10^{-2} \sim$ $10^{-4}$ is efficient and correction should be done after $6 \sim 30$ iterations. Correcting is especially useful after a significant increase (10 50\% depending on the grid size and solver) of the residual within three successive iterations, resulting in great acceleration of the speed of convergence for the following criteria in $l_{2}$ norm:

(a) $\left\|\mathbf{r}^{(N)}\right\| /\left\|\mathbf{r}^{(0)}\right\|<\varepsilon$ and $\sqrt{\left\langle\mathbf{M}^{-1} \mathbf{r}^{(N)}, \mathbf{r}^{(N)}\right\rangle} /\left\|\mathbf{r}_{\mathbf{o}}\right\|<\varepsilon$ for the relative residual,

(b) $D \cdot\left\|\operatorname{div} \mathbf{j}^{(N+1)}\right\| /\left\|\mathbf{j}^{(N)}\right\|<\varepsilon$ for the total current $\mathbf{j}^{(N)}$ ( $D$ is the average cell size),

(c) $\left\|\mathbf{E}^{(N), s}-\mathbf{E}^{(N-1), s}\right\| /\left\|\mathbf{E}^{(N), s}\right\|<\varepsilon$ for the numerical solution $\mathbf{E}^{(N), s}$.

Numerical results can be found in the papers of Smith (1996), Varentsov (1999) and Fomenko (1999). Maximum acceleration (up to 100 times) was achieved at low frequencies.

\subsection{The essential features of the algorithm}

The advantages of the algorithm presented here are summarized as follows:

(a) The matrix $\hat{\mathbf{A}}$ is complex symmetric and sparse, the algorithm requires very economical computer memory. Matrix $\hat{\mathbf{A}}$ has 13 bands. We need not store in the memory the eight bands in blocks $\mathbf{A}_{x y}, \mathbf{A}_{x z}, \mathbf{A}_{y x}, \mathbf{A}_{y z}, \mathbf{A}_{z x}, \mathbf{A}_{z y}$, because these bands contain only short arrays of grid cells; $\Delta_{i-1}^{\prime}$, $\Delta_{j-1}^{\prime}, \Delta_{k-1}^{\prime}$. Other blocks $\left(\mathbf{A}_{x x}, \mathbf{A}_{y y}, \mathbf{A}_{z z}\right)$ have five bands, but we need to store only three bands in the memory due to the complex symmetry of these blocks. Moreover, only the diagonal is complex, others bands are real.

FD algorithms for the magnetic field (Mackie et al., 1993; Varentsov, 1999) require the storing of at least seven bands in the memory (matrix has 13 bands). FE methods have at least 27 bands and require 14 bands to be stored (Xiong et al., 1999).

(b) Our choice of staggered grid nodes (Fig. 1) allows us to simplify the formulation of the boundary conditions. We use only two components of the electric field at the boundary planes. These components are tangential and continuous, so we do not need values of the normal component of the electric field.

(c) The effective ILU preconditioning of the ill-conditioned matrix essentially decreases the condition number in comparison with more simple preconditioning procedures (Alumbaugh et al., 1996; Weidelt, 1999; Xiong et al., 1999; Spichak, 1992) providing with fast convergence, high accuracy and stable solution follows in a wide range of frequencies, even in conductivity contrasts of up to 500,000 times (Fomenko, 1999). A comparison of different preconditioners was made by Fomenko (1999) and Varentsov (1999).

(d) The correction of divergence-free current during the iteration process allows us to obtain highly accurate results in the air nodes and in a wide range of frequencies, and the correction greatly accelerates the speed of convergence at low frequencies. Numerical results were demonstrated by Smith (1996) and Fomenko (1999).

(e) The proposed FD algorithm allows effective modeling at irregular staggered grids in comparison with IE methods that mainly use regular grids. The numerical results for the $3 \mathrm{~d} 2$ COMMEMI model in the next section demonstrate the high accuracy at irregular grids, which is no less than the accuracy of IE methods. The usage of irregular grids allows us to decrease the number of equations and calculation time, because we can only take small cell sizes near the conductivity breaks.

\section{Numerical Examples}

The validity of the proposed algorithm was checked using several models simulated with independent methods.

\subsection{Methodical research}

To demonstrate the essential features and effectiveness of the algorithm, we will show some methodical calculations. These calculations were made for the $3 \mathrm{~d} 2$ COMMEMI model (Fig. 2), i.e. one of the 3D models of the Comparison of Modeling in Electromagnetic Induction (COMMEMI) project (Zhdanov et al., 1997).

The $3 \mathrm{~d} 2$ COMMEMI model consists of two adjacent 1 and $100 \Omega \mathrm{m}$ blocks of dimensions $X: 20 \times Y: 40 \times$ $Z: 10 \mathrm{~km}$. The normal section of the model consists of three layers with resistivities of 10,100 , and $0.1 \Omega \mathrm{m}$. The upper two layers are 10 and $20 \mathrm{~km}$ thick, respectively. The conductivity contrast ratio in this model is up to 1000 and the main difficulty for the calculations is the presence of a strong resistivity contrast at the surface.

The irregular grid was used in this study. The cell size in the central part of the anomalous bodies was $X: 2 \times Y$ : $2 \times Z: 0.84 \mathrm{~km}$. The smaller cells were taken to be near the adjacent planes and the cell sizes extended to large distances from the blocks. The difference between the maximum and minimum size of the grid cell was two orders, the model was composed of $32 \times 28 \times 24=21,504$ cells. The condition number of the linear system in (9) was more than $10^{10}$. The computations were made at a period of $100 \mathrm{sec}$, and used the double precision on the PC (CPU: Pentium-III $500 \mathrm{MHz}$ ).

Figure 2(a) demonstrates the effectiveness of different solvers. The normalized residual $\|\mathbf{r}\| /\left\|\mathbf{r}_{\mathbf{o}}\right\|$ and $|\operatorname{div} \mathbf{J}| /$ $\left|\operatorname{div} \mathbf{J}_{0}\right|$ have shown fast quasi-monotonic convergence as geometrical series (almost a straight line in log 10-scale) for the QMRSTAB and BICGSTAB solvers (three-step solvers). 
(a)
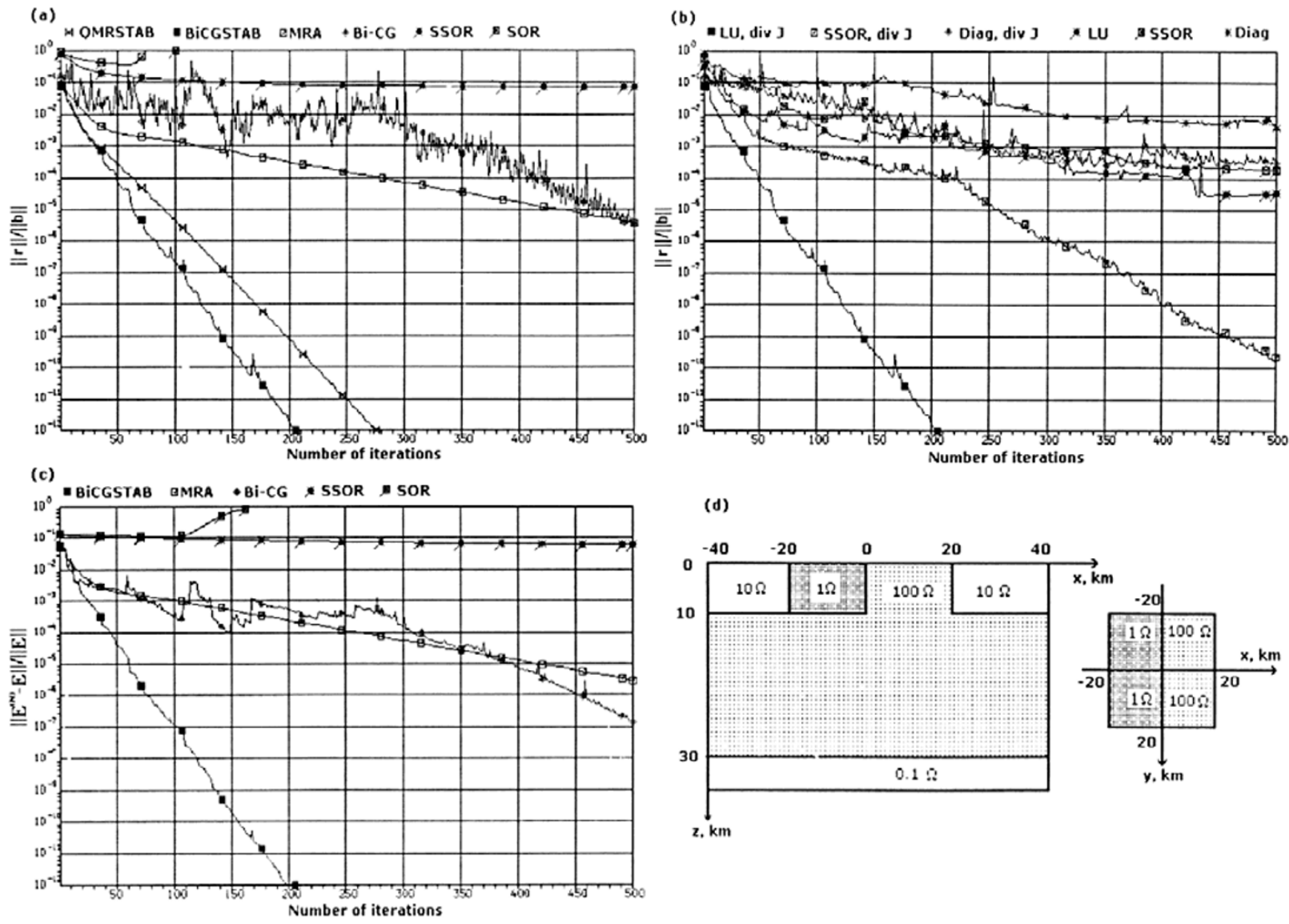

(d)

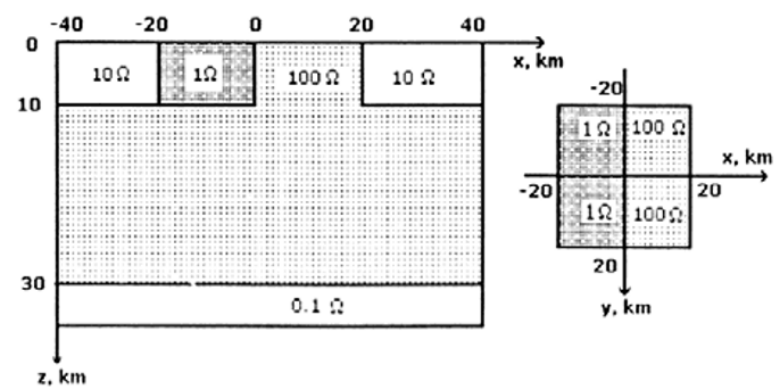

Fig. 2. The $3 \mathrm{~d} 2$ COMMEMI model simulation at $100 \mathrm{sec} ., E_{x}$ polarization. The panel (a) demonstrates the effectiveness of different solvers. Panel (b) shows the significance of using the div $\mathbf{J}$ correction procedure. The effectiveness of the different preconditioners (LU, SSOR and Diag.) is shown in panel (b). Panel (c) illustrates the convergence of an iterative solution to the reference solution for different solvers.

BICGSTAB is faster but shows more oscillations. It provide high-accuracy solutions (up to 12 order) within 200 iterations and less than $1 \%$ accuracy in 20 iterations. The second group of solvers (MRA, BICG : two-step solvers, see Mackie et al., 1993 and Smith, 1996) showed essentially slower convergence which was not so smooth. The third group: SSOR, SOR (see Spichak, 1992) could not calculate the $3 \mathrm{~d} 2$ model effectively at the irregular grid. The SSOR showed very slow convergence and practically stopped at $10 \%$ accuracy both for the residual and for the E-solution (Figs. 2(a) and 2(c)), and the SOR does not provide convergence at all.

We also investigated preconditioned normalized residual $\sqrt{\left\langle M^{-1} \mathbf{r}, \mathbf{r}\right\rangle} /\left\|\mathbf{r}_{\mathbf{o}}\right\|$, relative error for the secondary field $\left\|\mathbf{E}^{n+1}-\mathbf{E}^{n}\right\| /\left\|\mathbf{E}^{n+1}\right\|$, and normalized error for the reference solution $\left\|\mathbf{E}^{n}-\mathbf{E}^{\mathrm{ref}}\right\| /\left\|\mathbf{E}^{\mathrm{ref}}\right\|$ at each iteration. All of them have the same quasi-monotonic behavior, thus the first and second ones are not shown here. The illustration of convergence to the reference solution is shown in Fig. 2(c). The normalized error $\left\|\mathbf{E}^{n}-\mathbf{E}^{\text {ref }}\right\| /\left\|\mathbf{E}^{\text {ref }}\right\|$ also has quasimonotonic behavior. The reference solution was calculated with the best accuracy model (IG6 grid, see Table 1) by using the termination criterion shown in (17) for $\varepsilon=10^{-10}$. Our numerous calculations showed that obtaining each next order of accuracy depend only slightly on the period and size of matrix when we use the best three-step solvers, in contrast to the second and third group of solvers.

Figure 2(b) shows the effectiveness of different preconditioning schemes and the importance of the $\operatorname{div} \mathbf{J}$ correction procedure. The lines ' $L U$ ', 'SSOR' and 'Diag' demonstrate convergence for the different preconditioners without $\operatorname{div} \mathbf{J}$ correction, and the lines ' $L U, \operatorname{div} J$ ', 'SSOR, $\operatorname{div} J$ ' and 'Diag, $\operatorname{div} J$ ' show convergence when the $\operatorname{div} \mathbf{J}$ correction procedure is used. It is obvious that the $\operatorname{div} \mathbf{J}$ correction procedure greatly accelerates convergence. The LU preconditioner is several times faster than the SSOR and 20-100 times faster than the simple diagonal preconditioner (depending on the grid size, frequency, and model). The diagonal preconditioning (Diag) is very rough for ill-conditioned systems, it may require thousands of iterations to obtain $1 \%$ accuracy (Alumbaugh et al., 1996; Xiong et al., 1999).

Our numerical experiments at different periods confirmed the theoretical expectations discussed in Subsection 2.7. The maximum acceleration of the convergence (up to 100 times) was obtained at low frequencies due to $\operatorname{div} \mathbf{J}$ correction, similar to the results of Smith (1996).

Next, we carried out methodical research into the accuracy for using quasi-homogeneous and irregular grids. Some of the results are shown in Fig. 3. Three quasihomogeneous grids (HG) and six irregular grids (IG) were constructed. Quasi-homogeneous grids used equal cells inside the anomalous bodies for the $X, Y$ directions and ex- 

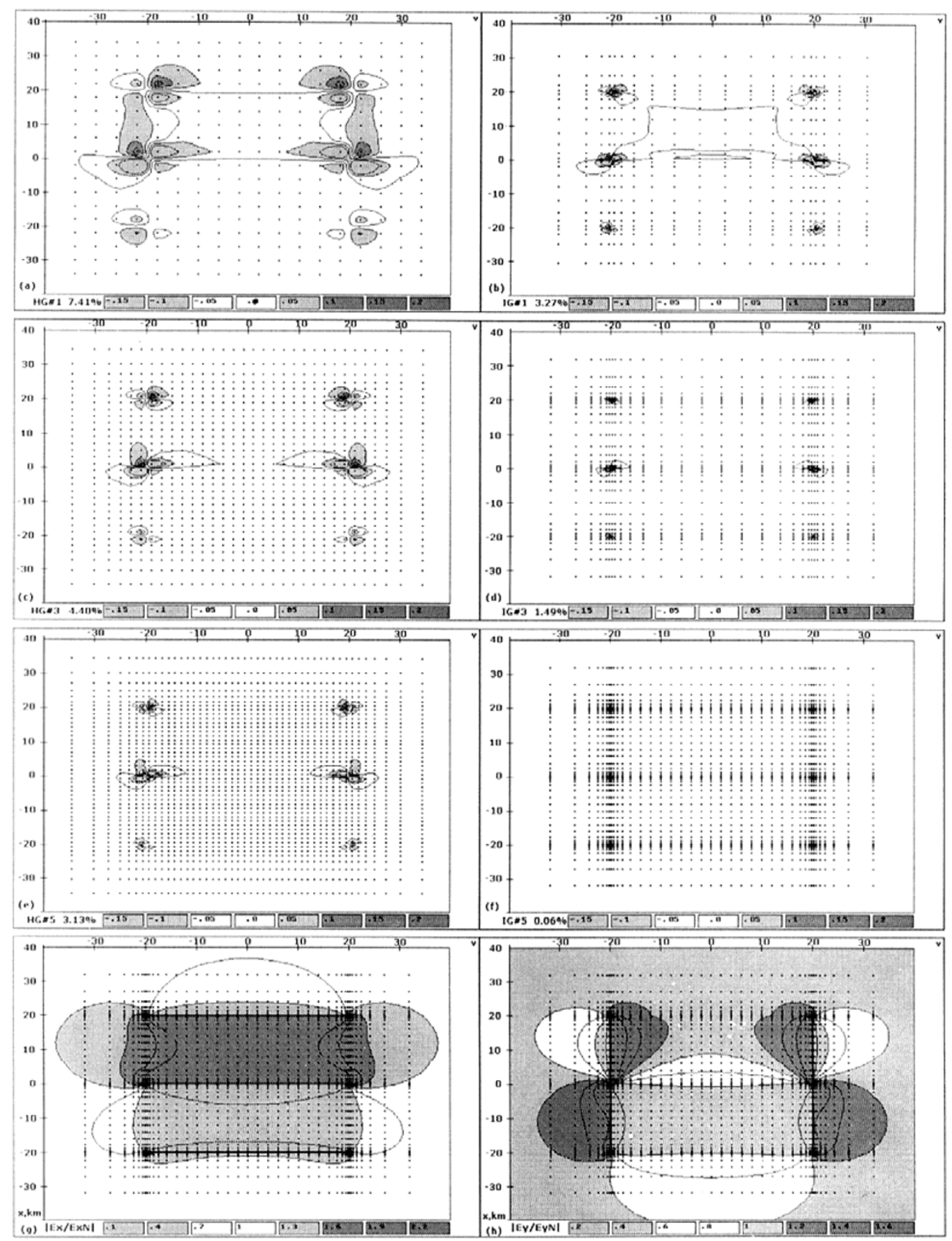

Fig. 3. The COMMEMI $3 \mathrm{~d} 2$ model simulation at $100 \mathrm{sec}$. The convergence at the sequence of quasi-homogeneous and irregular grids to the reference solution. Panels (g) and (h) show normalized amplitudes of $E_{x} / E_{x}^{N}$ and $E_{y} / E_{y}^{N}$ components for $E_{x}$ and $E_{y}$ polarization, respectively. The left panels (a), (c), (e) show the difference between solutions at a given grid and reference solution $\left\|\mathbf{E}_{x}-\mathbf{E}_{x}^{\text {ref }}\right\| /\left\|\mathbf{E}_{x}^{\text {ref }}\right\|$ at the homogeneous grids HG, right panels, (b), (d), (f) for the irregular grids IG ( $E_{x}$ polarization).

tended the cell size to outside the bodies. The irregular grids used small cells near conductivity breaks, thus providing better approximation in these areas. The 3D modeling area was $X:[-165 \mathrm{~km}, 165 \mathrm{~km}], Y:[-165 \mathrm{~km}, 165 \mathrm{~km}]$, $Z:[-100 \mathrm{~km}, 40 \mathrm{~km}]$, and the extent of drawing the results $X:[-40 \mathrm{~km}, 40 \mathrm{~km}], Y:[-40 \mathrm{~km}, 40 \mathrm{~km}]$ at the surface $z=0$. The solution at the best grid $I G 6$ was taken as the reference solution and it was calculated at the best accuracy level using the termination criterion shown in (17) for $\varepsilon=10^{-10} \%$. Solutions at the other grids were calcu- lated with $\varepsilon=0.1 \%$ level of reliable practical solution. A PC with Pentium-III, $500 \mathrm{MHz}$ CPU was used for the computing. Run time for grids of different sizes are shown in Table 1. Table 1 also shows that irregular grids allow one to obtain more accurate solutions, especially in the vicinity of conductivity break area, using fewer cells and fewer iterations. The misfits between the amplitude of $E_{x}$ for the solution at each grid and the reference solution are shown in Fig. 3 for the $E_{x}$ polarization case, at a period of $100 \mathrm{sec}$. 
Table 1. The convergence condition for various quasi-homogeneous and irregular grids. Model: 3d2 COMMEMI, computing period at 100 sec. $E_{x}, H_{y}$ were computing at the $E_{x}$ polarization case and $E_{y}, H_{x}$ were computed at the $E_{y}$ polarization case. Error was estimated as $\delta E_{x}(\%)=\left\|E_{x}^{\mathrm{ref}}-E_{x}\right\| /\left\|E_{x}^{\mathrm{ref}}\right\| \cdot 100$. The computing used BiCGSTAB as the solver and terminated at $\varepsilon=0.1 \%$ by using the termination criteria shown in (17). The run-time shown in this table is the case using a PC with Pentium III/500 MHz CPU.

\begin{tabular}{ccccccccrrr}
\hline Grid & $\begin{array}{c}\text { Number } \\
\text { of cells }\end{array}$ & $\begin{array}{r}\text { Number } \\
\text { of equat. }\end{array}$ & $\begin{array}{c}\text { Aver. cell } \\
\text { size }(\mathrm{km})\end{array}$ & $\begin{array}{r}\delta E_{x} \\
(\%)\end{array}$ & $\begin{array}{r}\delta E_{y} \\
(\%)\end{array}$ & $\begin{array}{r}\delta H_{x} \\
(\%)\end{array}$ & $\begin{array}{r}\delta H_{y} \\
(\%)\end{array}$ & $\begin{array}{r}\text { Number of } \\
\text { iterations }\end{array}$ & $\begin{array}{r}\text { Run-time } \\
(\mathrm{sec})\end{array}$ \\
\hline HG1 & 282815 & 55,854 & 2.99 & 7.41 & 15.2 & 12.7 & 16.7 & 70 & 75 \\
HG3 & 444420 & 165,555 & 2.52 & 4.40 & 9.28 & 5.14 & 8.55 & 120 & 371 \\
HG5 & 585827 & 357,894 & 1.59 & 3.13 & 6.60 & 5.46 & 7.22 & 192 & 1352 \\
IG1 & 343018 & 78,640 & 0.99 & 3.27 & 6.57 & 2.92 & 3.54 & 96 & 135 \\
IG2 & 383422 & 103,181 & 2.18 & 3.04 & 6.48 & 3.14 & 3.37 & 96 & 177 \\
IG3 & 443822 & 153,675 & 2.18 & 1.49 & 2.96 & 1.48 & 1.58 & 110 & 316 \\
IG4 & 524422 & 209,325 & 1.56 & 0.90 & 1.53 & 1.34 & 1.58 & 165 & 571 \\
IG5 & 605324 & 319,017 & 1.34 & 0.06 & 0.04 & 0.05 & 0.10 & 168 & 954 \\
IG6 & 605327 & 348,318 & 1.06 & & & & & 210 & 1355 \\
\hline
\end{tabular}
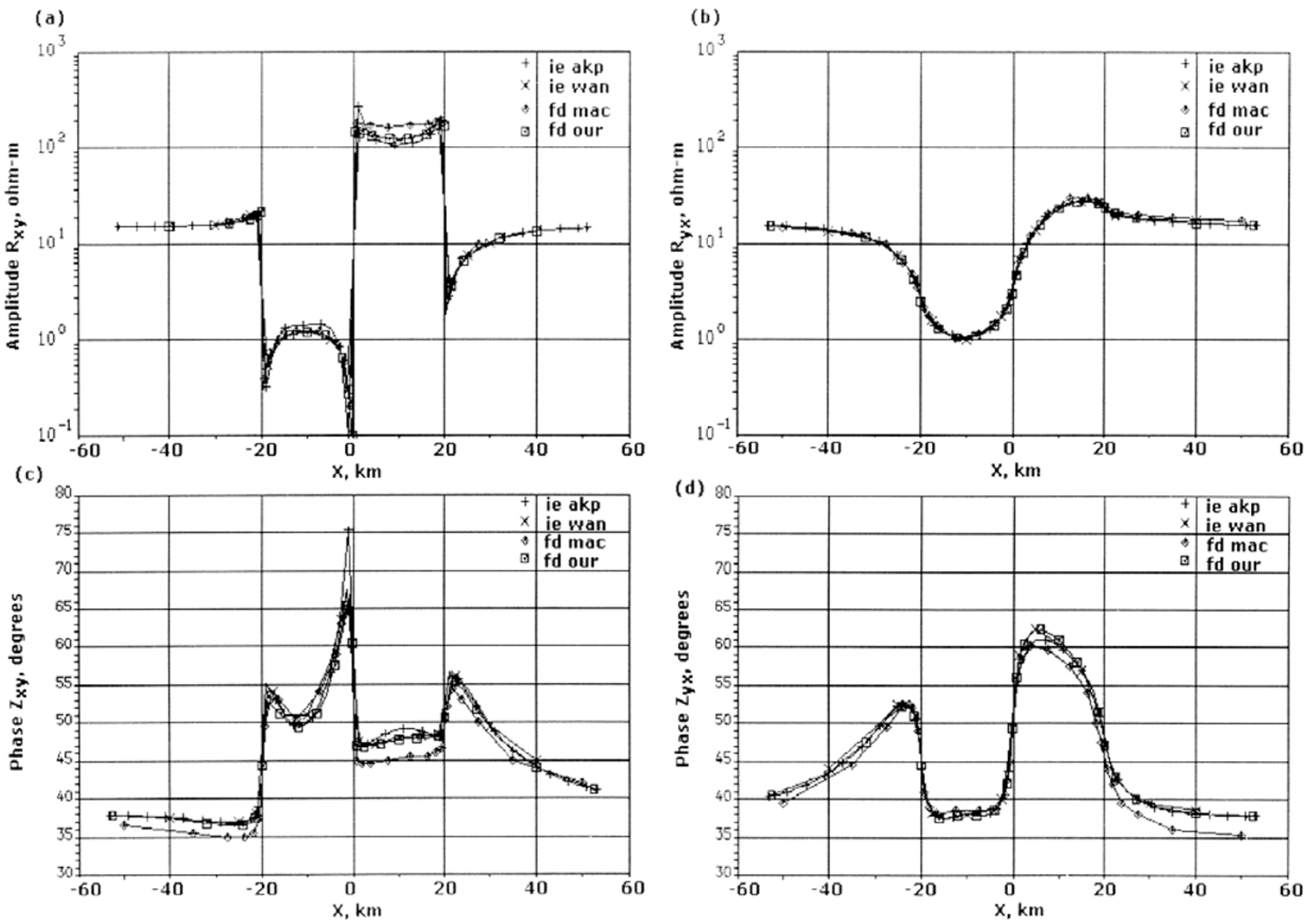

Fig. 4. The 3d2 COMMEMI model simulation at $100 \mathrm{sec}$. for $E_{x}$ polarization. Panels (a) and (b) are apparent resistivities $R_{x y}$ and $R_{y x}$, respectively, while panels (c) and (d) are phases of impedances $Z_{x y}$ and $Z_{y x}$. The comparison are between results from Avdeev et al. (1997) IE code-"ie akp", Wannamaker (1991) IE code-“ie wan", Mackie et al. (1993) FD code-“fd mac" and our FD code in this study-"fd our".

\subsection{Comparison for 3d2 COMMEMI model}

We calculated MT responses at a period of $100 \mathrm{sec}$. and compared our results with the IE solution of Avdeev et al. (1997), the IE solution of Wannamaker (1991), and the FD solution of Mackie et al. (1993). The results of Avdeev et al. (1997) were obtained directly from the authors, other results were redrawn from the figures of Mackie et al. (1993).

Our result used the irregular IG6 grid composed of $32 \times$ $28 \times 24=21,504$ cells (see Subsection 3.1), where the average size of the cell is of a volume of $1.06 \mathrm{~km}^{3}$, and $X$ : $2 \times Y: 2 \times Z: 0.84 \mathrm{~km}$ cells. The cell in the central part of the blocks and small cells up to $X: 0.2 \times Y: 0.2 \times Z: 0.15$ 
$\mathrm{km}$, are near the boundaries. The run time for $\varepsilon=0.01 \%$ in stop-criteria (17) was $88 \mathrm{sec}$. on a PC with Pentium-II/400 $\mathrm{MHz}$. Avdeev et al. (1997) used a homogeneous grid inside the block with horizontal dimensions of $X: 1 \times Y: 1 \mathrm{~km}$ and with vertical dimensions of $Z: 1,1.5,2,2.5$ and $3 \mathrm{~km}$ in the block area. The run time for $40 \times 40 \times 5=8,000$ cells using a Pentium/586 PC was approximately 5 min. Mackie et al. (1993) used a rougher irregular grid with $X: 5 \times Y: 5$ $\mathrm{km}$ horizontal dimensions in the central part of the blocks $(28 \times 28$ cells on XOY plane $)$ and $X: 1.25 \times Y: 1.25 \mathrm{~km}$ cells near the conductivity contrasts. The run time was 25 min for Mackie's code using a CRAY-2/4-256 computer.

Figure 4 shows the apparent resistivities $R_{x y}, R_{y x}$ and phases of impedance $Z_{x y}, Z_{y x}$ along the central profile $y=$ 0 . In general, all results showed good agreement between the curves. Our results are closest to the IE solution of Wannamaker (1991). In spite of using a horizontal cell size in the central part of the blocks that was twice larger than that of Avdeev et al. (1997), we only had small misfits with their results, because we used the irregular grid. Some visible differences were seen between our results, the IE solutions (Wannamaker, 1991; Avdeev et al., 1997) and the Mackie et al. (1993) FD results. We explain this by using a rough grid in the central part of the blocks of Mackie et al.

The size of the grid is very important for the accuracy, as shown in the comprehensive comparison of the $3 \mathrm{~d} 2$ COMMEMI model and two other models (Varentsov et al., 2000). Four algorithms; two IE methods (Wannamaker, 1991; Xiong et al., 1999) and two FD methods (Varentsov, 1999; Fomenko, 1999) were compared. This comparison used a modern powerful PC and large grids with smaller cells than the former COMMEMI-I project (Zhdanov et al., 1997). In particular, this new COMMEMI comparison showed that the size of the grid essentially influences the approximation and accuracy of the calculation of $E$ and $H$ components. Mackie et al. (1993) used a grid with the average size as used in COMMEMI-I. Our FD method here, Avdeev et al. (1997) and Wannamaker (1991) used grids with smaller cells, so we obtained good coincidence between all of us and a visible difference from Mackie et al. (1993). The referenced COMMEMI report (Varentsov et al., 2000) contains many comparative results.

\subsection{D topography model}

Topography is represented as an anomaly in the air layer in our program. To do this, we should overcome the problem of a large resistivity contrast between the air layer and the ground. We selected a 2D-topography model 'trapezoidal hill' (Fig. 5) to check the validity of our method for a resistivity contrast $10^{6}$ times between air and the hill. Conductivities of the host and the hill have $10^{-2} \mathrm{~S} / \mathrm{m}$, air has $10^{-8} \mathrm{~S} / \mathrm{m}$, and the frequency is $2 \mathrm{~Hz}$. In the hill area, we used $X: 50 \times Y: 100 \times Z: 28 \mathrm{~m}$ cells (the hill consists of 16 rows of cells). Outside the hill, irregular cells were used. The ratio between the maximum and minimum size of the grid cell was 90 . The total number of nodes were $64 \times 25 \times 38=60,800$.

We compared the calculated apparent resistivity $R_{x y}$ and $R_{y x}$ with the FD results of Ngoc (1980) and the FE results by Wannamaker et al. (1986). One percent accuracy for the normalized residual $\|\mathbf{r}\| /\left\|\mathbf{r}_{\mathbf{0}}\right\|$ is achieved after 43 iterations

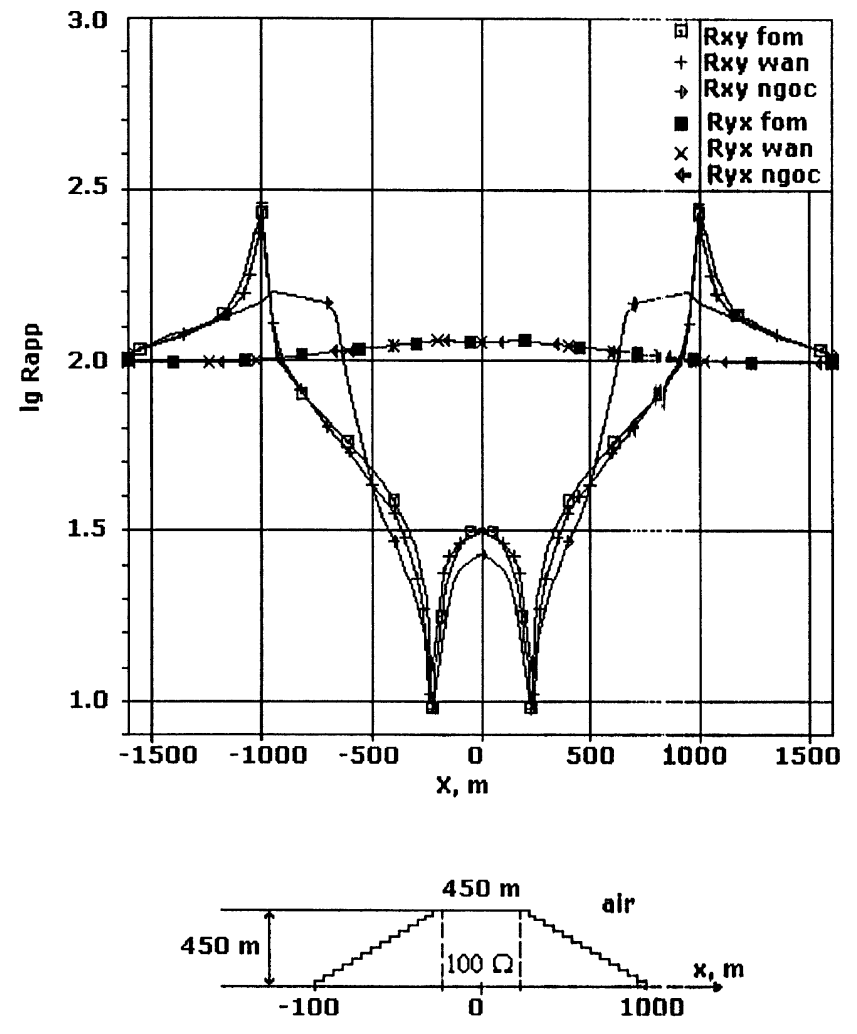

Fig. 5. 2D MT simulation of a trapezoidal hill at $2 \mathrm{~Hz}$. Our FD results, 'Rxy fom' and 'Ryx fom', are compared with the FD results of 'Rxy ngoc', 'Ryx ngoc' (Ngoc, 1980) and FE results of 'Rxy wan' and 'Ryx wan' (Wannamaker et al., 1986). The hill is composed of 16 grids in the vertical direction and each cell size is $X: 50 \times Y: 100 \times Z: 28 \mathrm{~m}$.

in the $E_{x}$ polarization (TM mode) and 72 iterations in the $E_{y}$ polarization (TE mode), and it required 115 and $190 \mathrm{sec}$. of computing time, respectively, using a PC with Pentium $166 \mathrm{MHz}$ CPU. The H-field was calculated as described in Subsection 2.6. The $\mathbf{E}$ and $\mathbf{H}$ fields were computed at the center of the rectangular cell faces $x_{i}^{\prime}, y_{j}^{\prime}$ at each level $z=z_{k}$. Our results agree well with the FE solution of Wannamaker et al. (1986), for both TM $\left(R_{x y}\right)$ and TE $\left(R_{y x}\right)$ modes. Ours and the FE solution, however, have visible differences from the FD solution of Ngoc (1980) for $R_{x y}$, but have good coincidence for $R_{y x}$.

\subsection{Model with topography and water reservoir}

We considered a model that studies the interaction of the topography with subsurface structures such as a low resistivity water reservoir. The topography is represented by a local 3D hill of $500 \mathrm{~m}$ width in the $x$ direction, $200 \mathrm{~m}$ in the $y$ direction and a height of $50 \mathrm{~m}$ (Fig. 6). The hill is formed by the cells with conductivity $\sigma=10^{-2} \mathrm{~S} / \mathrm{m}$. The first layer is $300 \mathrm{~m}$ thick and $\sigma_{1}=10^{-2} \mathrm{~S} / \mathrm{m}$, the second layer (host) $-\sigma_{2}=10^{-3} \mathrm{~S} / \mathrm{m} ; \sigma_{\text {air }}=10^{-8} \mathrm{~S} / \mathrm{m}$.

There are two water reservoirs with conductivity $\sigma_{\text {water }}=$ 1.0 S/m under the hill. Their sizes are shown in Fig. 5. The left reservoir is represented by $4 \times 4 \times 4$ cells, the right reservoir by $4 \times 4 \times 2$ cells. The model is composed of $37 \times 26 \times 41=39,442$ cells. One percent accuracy for $\|\mathbf{r}\| /\left\|\mathbf{r}_{\mathbf{0}}\right\|$ was obtained within 19 iterations in the $E_{x}$ polarization case and nine iterations in the $E_{y}$ polarization case using a BICGSTAB solver, and the calculation time 
(a)

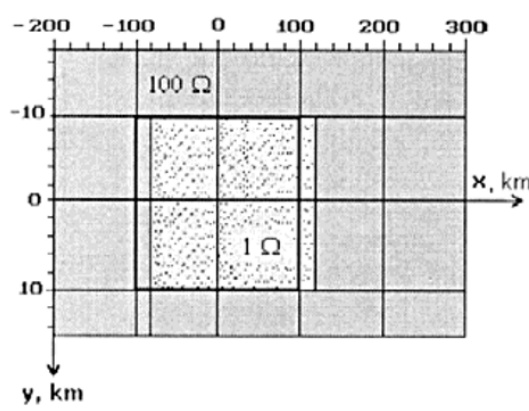

(c)

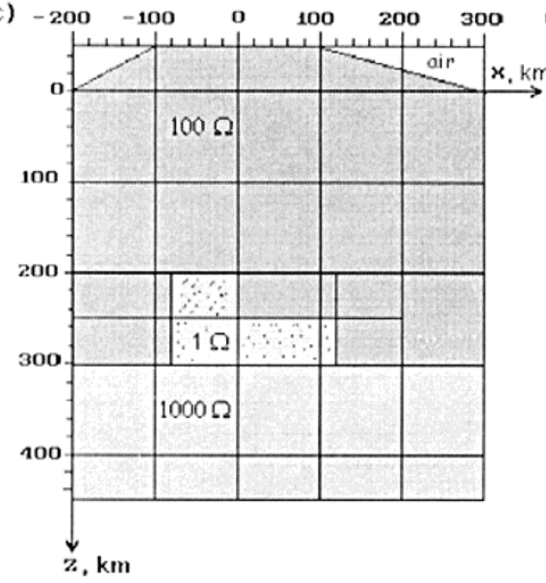

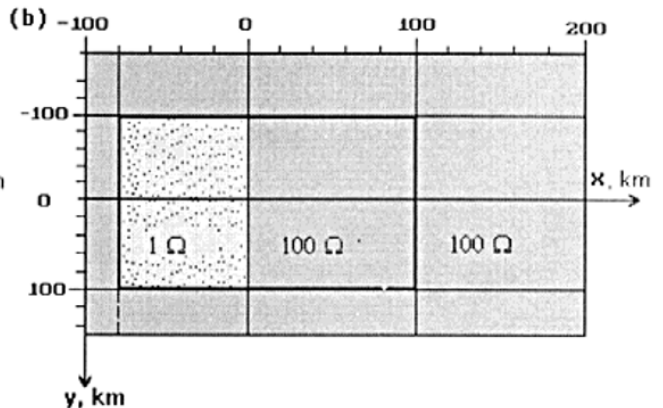

(d) -100

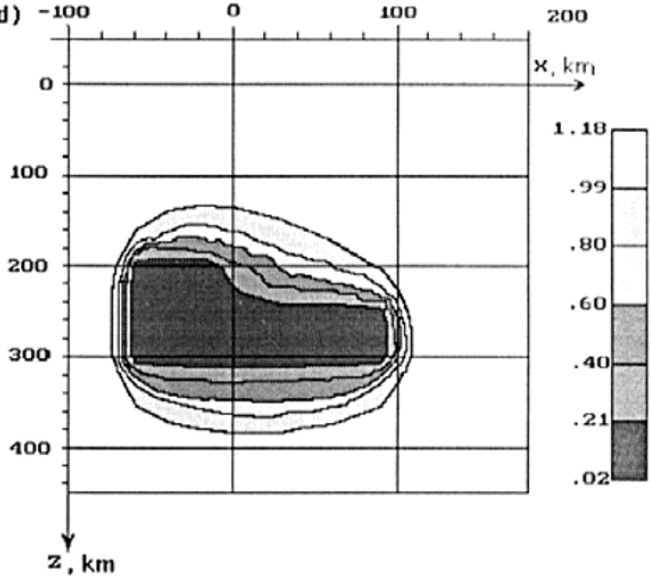

Fig. 6. The MT simulation of water reservoir with a topography model computing at $10 \mathrm{~Hz}$. The upper panels show the XY section of the model, panel (b) at $z=200 \mathrm{~m}$ and panel (a) at $z=250 \mathrm{~m}$, bold contours show the perimeter of the hill at $z=50 \mathrm{~m}$. Panel (c) shows the XOZ section of the model at $y=0$. Panel (d) shows the calculated normalized electric field amplitude $\left|\mathbf{E}_{x} / \mathbf{E}_{x}^{p}\right|$ for $E_{x}$ polarization.

required $105 \mathrm{sec}$. using a PC (Pentium $166 \mathrm{MHz} \mathrm{CPU})$. The normalized amplitude of the electric field $\left|E_{x} / E_{x}^{p}\right|$ for the $E_{x}$ polarization case is shown in Fig. 6. The results of $3 \mathrm{D}$ modeling accurately represent the water reservoir contour in the XOZ plane in spite of using a rough grid with only a few cells for each body and only two cells in the $Z$ direction for the second body. The value of $E_{x} / E_{x}^{p}$ falls five times inside the water reservoir.

\section{Conclusions}

The fast, accurate, and economical staggered grid finite difference method is developed for calculating 3D conservative electromagnetic fields in $3 \mathrm{D}$ media with topography and high resistivity contrasts over a wide range of frequencies. It has a good choice of staggered grids, a simple but effective preconditioner, support of divergence-free current, and powerful CG solvers. The proposed scheme is economical: computer memory requirements depend linearly on the number of grid cells because the matrix of the linear system is banded. Most of the applied problems can be solved with a PC of 64-128 MB memory. The algorithm has obtained $1 \%$ accuracy with quasi-monotonic convergence of normalized residual within the first ten iterations using grids of moderate size $(30,000-100,000$ cells), and within hundred of iterations for extremely high accuracy. The accuracy of our FD code is comparable with the accuracy of IE algorithms. The effective preconditioning allows highaccuracy results using irregular grids, which decreases the number of cells and calculation time, because we can take small cells only near the conductivity breaks. The method is effectively applicable to models with large resistivity contrasts and complicated topography.

Due to the high accuracy of wide-band frequencies (up to 10 decades), the method is applicable for computing a time-domain response. Another additional advantage of the presented method is that the conductivity distribution is concentrated only at the diagonal of the matrix. Because of this, the approach is attractive for use in inverse problems.

Acknowledgments. This research was supported by JCR Co. Ltd., JMC Geothermal Engineering Co. Ltd., and RFBR grant 98-0565411. The authors thank Dmitry Avdeev, Alexey Kuvshinov, and Oleg Pankratov for providing numerical results for comparison. We are also grateful to Ivan Varentsov and Vjacheslav Spichak for many fruitful discussions during this study. We also thank our reviewers, Philip Wannamaker and Yasuo Ogawa, for their detailed comments, which helped us to improve this paper.

\section{References}

Alumbaugh, D. L., G. A. Newmann, L. Prevost, and J. Shadid, Threedimensional wide-band electromagnetic modeling on massively parallel computers, Radio Sci., 31, 1-23, 1996.

Avdeev, D. B., A. V. Kuvshinov, O. V. Pankratov, et al., High performance three dimensional electromagnetic modeling using modified Neumann series. Wide-band numerical solution and examples, J. Geomag. Geoelectr., 49, 1519-1539, 1997.

Chan, T. F., E. Gallopoulos, V. Simoncini, et al., A quasi-minimal residual variant of the Bi-CGSTAB algorithm for non-symmetric systems, SIAM J. Sci. Statist. Comput., 15, 338-347, 1994.

Fomenko, E. Yu., MT and control source modeling algorithms for 3D media with topography and large resistivity contrasts, in The Second International Symposium on Three-Dimensional Electromagnetics (3DEM-2), Salt Lake City, Utah, USA. Extended Abstracts, edited by P. Wannamaker and M. Zhdanov, pp. 21-24, 1999. 
Harrington, R. F., Time-harmonic electromagnetic fields, McGraw-Hill Book Co., 1961.

Mackie, R. L., T. R. Madden, and P. E. Wannamaker, Three-dimensional magnetotelluric modeling using finite difference equations-Theory and comparisons to integral equation solutions, Geophysics, 58, 215-226, 1993.

Manteuffel, T. A., G. Starke, and R. S. Varga, Adaptive k-step iterative methods for non-symmetric systems of linear equations, Electron. Trans. on Num. Analysis., 3, 50-65, 1995.

Ngoc, P. V., Magnetotelluric survey of the mount Meager region of the Squamish Valley (British Columbia), Geomagnetic Service of Canada, Earth Physics Branch of the Dept. of Energy, Mines and Resources Canada, OF Rep., 80-8-E, 1980.

Sasaki, Y., Three-Dimensinal Frequency-Domain Electromagnetic Modeling Using the Finite-Difference Method, Buturi-Tansa (Geophysical Exploration), 52, 421-431, 1999 (in Japanese with English abstract and figure captions).

Smith, J. T., Conservative modelling of 3-D electromagnetic fields, Geophysics, 61, 1308-1324, 1996.

Spichak, V. V., Differential boundary conditions for the electric and magnetic fields in the infinite conductive media, Electromagnetic Sounding of the Earth, Moscow, IZMIRAN, 13-21, 1985 (in Russian).

Spichak, V. V., Mathematical modeling of EM fields in 3D inhomogeneous media, 191 pp., Moscow, Nauka, 1992 (in Russian).

Steijpen, G. L. G., H. A. Van der Vorst, and D. R. Fokkema, BICGSTAB(1) and other hybrid BiCG methods, Num. Algorithms, 7, 75-109, 1994.

Van der Vorst, H. A., BI-CGSTAB: a fast and smoothly convergent variant of Bi-CG for the solution of non-symmetric linear systems, SIAM J. Sci. Statist. Comput., 13, 631-644, 1992.

Varentsov, Iv. M., The selection of effective finite difference solvers in 3D electromagnetics modeling, in The Second International Symposium on Three-Dimensional Electromagnetics (3DEM-2), Salt Lake City, Utah, USA, Extended Abstracts, edited by P. Wannamaker and M. Zhdanov, pp. 201-204, 1999.
Varentsov, Iv. M., E. Yu. Fomenko, N. G. Golubev, S. Mehanee, G. Hursan, and M. Zhdanov, Comparative study of 3-D finite-difference and integral equation methods, Annual Report of the Consortium for Electromagnetic Modeling and Inversion (CEMI), University of Utah, Salt Lake City, 401-440, 2000.

Wannamaker, P. E., Advances in modeling three-dimensional magnetotelluric responses using integral equations, Geophysics, 56, 1716-1728, 1991.

Wannamaker, P. E., J. A. Stodt, and L. Rijo, Two-dimensional topographic responses in magnetotellurics modeled using finite elements, Geophysics, 51, 2131-2144, 1986.

Weaver, J. T., A. K. Agarwal, and X. H. Pu, Recent developments in three-dimensional finite difference modelling of the magnetic field in geo-electromagnetic induction, in Three-Dimensional Electromagnetics, edited by M. Oristaglio and B. Spies, SEG, pp. 131-141, 1999.

Weidelt, P., Three-dimensional conductivity models: implications of electric anisotropy, in Three-Dimensional Electromagnetics, edited by $\mathrm{M}$. Oristaglio and B. Spies, SEG, pp. 119-137, 1999.

Xiong, Z., A. Raiche, and F. Sugeng, Efficient solutions of full domain 3D electromagnetic modelling problems, in The Second International Symposium on Three-Dimensional Electromagnetics (3DEM-2), Salt Lake City, Utah, USA, Extended Abstracts, edited by P. Wannamaker and M. Zhdanov, pp. 3-7, 1999.

Zhdanov, M. S., N. G. Golubev, V. V. Spichak, and Iv. M. Varentsov, The construction of effective methods for electromagnetic modeling, Geophys. J. R. Astr. Soc., 68, 589-607, 1982.

Zhdanov, M. S., Iv. M. Varentsov, J. T. Weaver, N. G. Golubev, and V. A. Krylov, Methods for modeling electromagnetic fields (results from COMMEMI), J. Appl. Geophys., 37, 133-271, 1997.

E. Yu. Fomenko (e-mail: efomenko@cs.msu.su) and T. Mogi (e-mail: tmogi@eos.hokudai.ac.jp) 